\title{
Enhanced Nonlinear Absorption Performance of Reduced Graphene Oxide Nanohybrid Covalently Functionalized by Porphyrin via 1,3-Dipolar Cycloaddition
}

\author{
Nan Zhang, Xiaoqing Cong* \\ School of Energy \& Power Engineering, Jiangsu University, Zhenjiang, China \\ Email: *cxq066@163.com
}

How to cite this paper: Zhang, N. and Cong, X.Q. (2018) Enhanced Nonlinear Absorption Performance of Reduced Graphene Oxide Nanohybrid Covalently Functionalized by Porphyrin via 1,3-Dipolar Cycloaddition. Materials Sciences and Applications, 9, 972-984.

https://doi.org/10.4236/msa.2018.912070

Received: October 15, 2018

Accepted: November 20, 2018

Published: November 23, 2018

Copyright $\odot 2018$ by authors and Scientific Research Publishing Inc. This work is licensed under the Creative

Commons Attribution International License (CC BY 4.0).

http://creativecommons.org/licenses/by/4.0/

\begin{abstract}
Porphyrin-functionalized reduced graphene oxide (RGO-TPP) was prepared by 1,3-dipolar cycloaddition reaction and characterized by Fourier transform infrared spectroscopy, Raman, ultraviolet/visible absorption, fluorescence, and transmission electron microscopy. At the same level of linear transmittance, RGO-TPP exhibited more enhanced optical nonlinearities than RGO and the pristineporphyrin, implying a remarkable accumulation effect as a result of the covalent link between RGO and porphyrin. The role of energy/electron transfer in the optical nonlinearities of RGO-TPP was investigated by fluorescence and Raman spectroscopy. All the results displayed that RGO can be covalently functionalized with porphyrins by the proposed approach.
\end{abstract}

\section{Keywords}

Reduced Graphene Oxide, Porphyrinoids, Cycloaddition Reaction, Nonlinear Optics

\section{Introduction}

In 2010, the Nobel Prize in Physics was awarded to Geim and Novoselov because of their work on the isolation of a free-standing monolayer sheet of graphene [1], which opened up a whole selection of properties on graphene ranging from enhanced electrical conductivity, mechanical strength, optical transparency and thermal conductivity, and so on [2] [3] [4]. Thanks to the extraordinary and superior properties, the grapheme has been acted as the building block of most 
graphitic materials and has attracted intense attention from both the experimental and the theoretical scientific worlds in recent years [5]. Just as with other allotropes of carbon, such as carbon nanotubes, the applications of graphene are limited by the poor solubility and processability as a result of the strong attractive van der Waals forces between grapheme nanosheets [6]. It is therefore very interesting to design and prepare graphene-based solution-processed organic/polymeric materials, due to its amenability to process in bulk-scale for the preparation of nanoscale hybrids, polymer blends and organic-inorganic nanocomposites, which are applicable in wide fields such as solar cells, optical sensors, and optical elements in lasers [7]. Remarkably, the hybrids are soluble enough to facilitate manipulation and solution studies in comparison with that of graphene [8]. Moreover, it can form donor-acceptor charge/electron-transfer complexes that bear great promise for major breakthroughs in converting solar energy into electricity, and also exhibit increased nonlinear optical (NLO) effects as potential optical limiting (OL) materials for the protection of human eyes and optical sensors [9].

Porphyrins, which have a large delocalized conjugated $\pi$-electron system spread over the macrocycle ring, are an interesting class of materials for NLO investigations that are characterized by its strong nonlinear absorption over a broad range of the visible spectrum, long lived excited state lifetimes, and its outstanding thermal and photostability and singular physical properties [10]. In addition, the design potential of these materials provides an opportunity for fine tuning the linear and NLO performances and the OL response of porphyrin-based materials [11]. These features render them exceptional donor/antenna building blocks. Incorporating them into donor-acceptor systems with, for example, graphene oxide, single-walled carbon nanotubes and multiwalled carbon nanotubes, gives rise to species that have not only the intrinsic properties of nanostructured carbon allotrope-based materials and porphyrins, but also some functions as a result of the mutual $\pi$ interaction between the carbon allotrope-based materials and porphyrins [12] [13].

In this paper, our interest is to develop a porphyrin-reduced graphene oxide nanohybrid (RGO-TPP) as a NLO material having an effective OL response. Unlike GO, which has chemical reactive oxygen-containing groups (carboxylic acid, epoxy and hydroxyl groups) at the edges or on the basal planes, and is a low electron/hole transporting material because of the disrupted sp2 bonding networks, the electron/hole transporting and electrical conductivity properties of RGO can be recovered following reduction and restoration of the $\pi$ network [14]. Importantly, organic moieties can be chemically grafted to the surface of RGO with retention of the structural integrity of the RGO frame work in which there is no loss of electronic structure [15]. Nevertheless, up to the present moment, the functionalized graphene reported in the literature mainly concerns the chemistry of GO due to the presence of oxygen functionalities [12], with fewer reports investigating covalently chemical modification of RGO, especially for 
those involving the investigation of optical nonlinearities of functionalized RGO systems [9] [14]. Encouraged by these considerations, we report, for the first time, the preparation of the porphyrin acrylates-modified RGO by 1,3-dipolar cycloaddition reaction, in which porphyrin units act as donor and RGO as acceptor. The result antRGO-TPP nanohybrid exhibit superior NLO effect compared to the separated RGO and porphyrin.

\section{Experimental Section}

\subsection{Materials and Methods}

All reagents were chemical or analytical grade and deionized water was used for all experiments. Graphite powder was purchased from Qingdao Zhongtian Co. Ltd. Graphene oxide (GO) was obtained from graphite according to the Hummers method [16], and dried for 6 days over $\mathrm{P}_{2} \mathrm{O}_{5}$ before use. RGO was prepared by the reduction of GO with $\mathrm{NaBH}_{4}$ as reductant in an oil bath at $80^{\circ} \mathrm{C}$ for $10 \mathrm{~h}$ [17]. TPP was prepared according to the literature [18].

Functionalization of RGO with TPP (RGO-TPP): The 1,3-dipolar cycloaddition reaction employed to prepare the porphyrin-functionalized RGO nanohybrid was performed under $\mathrm{N}_{2}$ atmosphere (Scheme 1). To a $50 \mathrm{~mL}$ three-necked glass reactor was added RGO (40 mg), TPP (35 mg), sarcosine (125 $\mathrm{mg}$ ) and DMF (20 mL). And then, the mixture was allowed to reflux for 5 days under a blanket of $\mathrm{N}_{2}$. After termination of reaction, the flask was allowed to cool to room temperature while stirring was maintained. The resultant material was precipitated by addition of water. The crude product was collected by filtration through a $0.45 \mu \mathrm{m}$ nylon membrane, washed with distilled water, dichloromethane and diethyl ether several times to remove excess TPP and other contaminants. The final product (RGO-TPP) was dried under vacuum at room temperature for $24 \mathrm{~h}$.

\subsection{Characterization}

Fourier transform infrared (FTIR) spectra were recorded on a MB154S-FTIR spectrometer (Canada) with wavenumber ranging from 400 to $4000 \mathrm{~cm}^{-1}$. The UV/vis absorption spectra of all samples in DMF were obtained on a JASCO

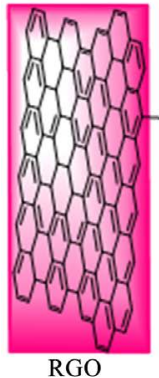

RGO

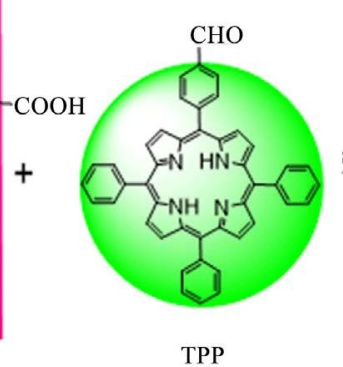

TPP

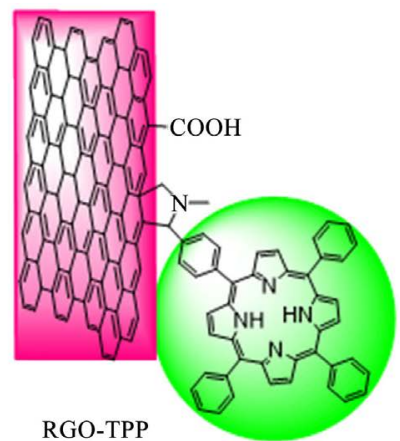

RGO-TPP

Scheme 1. Chemical route followed to functionalize RGO withporphyrin: Preparation of RGO-TPP nanohybrid. 
V-570 spectrophotometer at room temperature in the range between 200 and $700 \mathrm{~nm}$. Fluorescence spectra were taken on a Fluoro-Max-P Fluorescence Spectrophotometer. Raman spectra of the samples were performed at room temperature using a RenishawInvia Raman Microscope excited at a wavelength of $532 \mathrm{~nm}$. The morphology of the products was characterized by using a JEM-2100 (JEOL) Transmission Electron Microscope (TEM) working at $200 \mathrm{kV}$. Samples for TEM imaging were prepared by placing a drop of a dilute dispersion of the as-prepared products on amorphous carbon-coated copper grids and then drying in air before transfer to the TEM sample chamber.

\subsection{NLO Property Measurement}

The open-aperture Z-scan developed by Sheik Bahae et al. [19] was carried out by using a mode-locked Nd:YAG laser of $4 \mathrm{~ns}$ pulse length at $532 \mathrm{~nm}$ with a repetition rate of $2 \mathrm{~Hz}$. The laser beam was focused with a $40 \mathrm{~cm}$ focal length planoconvex lens. DMSO solutions of all samples were placed in a quartz cell of $2 \mathrm{~mm}$ thickness, which was controlled by a computer, and then moved along the $\mathrm{z}$-axis of the incident beam. Input fluence as well as the transmitted fluence was measured by two pyroelectric energy detectors (Rjp-765 energy probe), which areconnected to an energy meter (Rj-7620 ENERGY RATIOMETER, Laserprobe). A plot of normalized transmittance versus sample position provides information about optical nonlinearities of the sample. The reduction in transmittance recorded with open-aperture is independent of the nonlinear refraction and thus can be used to characterize the nonlinear absorption performance. For direct comparison, the linear transmittance of all samples was adjusted to $71 \%$ at $532 \mathrm{~nm}$.

\section{Results and Discussion}

\subsection{FTIR Analysis}

The evidence of successful functionalization following the covalent coupling of theporphyrinonto RGO via 1,3-dipolar cycloaddition reaction can be obtained from the FTIR spectra. As shown in Figure 1, the FTIR spectrum of RGO is almost featureless, and the typical absorption bands of GO nearly disappeared, which is in agreement with previous observations [20] [21] and with removal or transformation of much of the oxygen functionalities in $\mathrm{GO}$ on its conversion into RGO. After covalent functionalization with porphyrins, the characteristicporphyrin absorptions were observed [20] [22] [23]. The absorption band at around $3435 \mathrm{~cm}^{-1}$ can be ascribed to the stretching vibration of the pyrrole $\mathrm{N}-\mathrm{H}$ of the porphyrin. The peak appear at $1173 \mathrm{~cm}^{-1}$ corresponding to the stretching vibration of the pyrrole C-N bonds. A series of absorption bands from 1390 to $1700 \mathrm{~cm}^{-1}$ can be assigned to the stretching vibration of the phenyl $\mathrm{C}=\mathrm{C}$ bonds of the porphyrin. These observations are all clearly in agreement with the existence of porphyrins grafted to the RGO sheets. It should be noted that the interaction between RGO and porphyrin is not a simple physical adsorption, because the RGO-TPP nanohybrid was washed vigorously multiple times. 


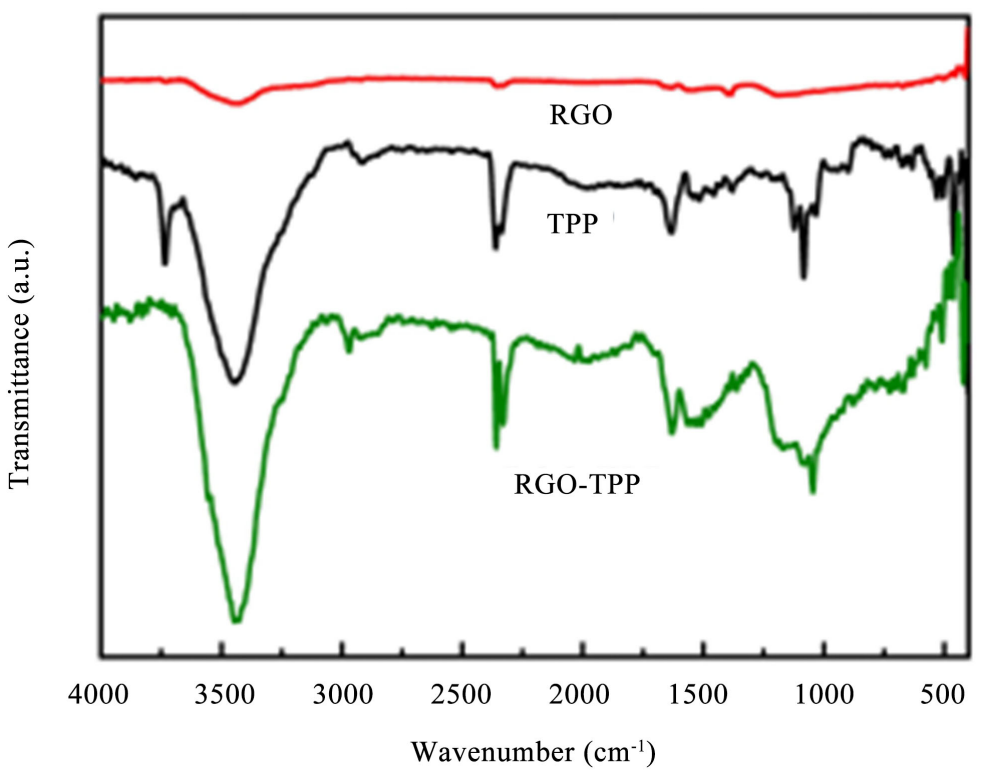

Figure 1. FTIR spectra of RGO, TPP and RGO-TPP.

\subsection{Linear Optical Absorption and Fluorescence Spectra Analysis}

The fingerprint of RGO-TPP nanohybrid is its electronic behavior; the UV/vis absorption and fluorescence spectra are sensitive to the presence of donor/acceptor groups, which can alter the optical properties by both charge and/or energy transfer [24]. With regard to the presence of RGO we are interested in using good solvent (such as DMF) for RGO dispersion to compare the electronic properties of RGO-TPP with those of pure porphyrin. The UV/vis absorption spectra of the samples are shown in Figure 2, where comparisons between pure porphyrinTPP and RGO-TPP hybrid can be seen. The electronic absorption spectrum of TPP is characterized by an intense Soret band at around $416 \mathrm{~nm}$, together with four relatively weaker Q bands between 500 and $700 \mathrm{~nm}$, which is in agreement with spectra of porphyrin analogues [25]. The RGO exhibits a broad absorption at $275 \mathrm{~nm}$ which is continuously decreasing to $800 \mathrm{~nm}$. The broad absorption observed at about $325 \mathrm{~nm}$ in the spectrum of RGO-TPP was ascribed to the $\pi$ - $\pi^{*}$ transition of highly delocalized $\pi$ electrons due to the interaction between RGO and porphyrin moieties. In addition, similar to the porphyrin functionalized single-walled carbon nanotubes and multi-walled carbon nanotubes in previous literatures [13] [26], the intensity of RGO-TPP was diminished in comparison with that of TPP. These UV/vis findings attest the success of the attachment of porphyrin molecules on RGO, and may well suggest a notable electronic interaction between porphyrin units and the RGO sheet in the ground state happens (porphyrin is usually regarded as the electron donor, whereas the graphene sheets are regarded as electron acceptors) [15].

More decisive tests about the interactions between the TPP and RGO constituents are based on fluorescence experiments, especially considering the prominent TPP fluorescence features. Photoexciting the TPP reference in the Soret band $(420 \mathrm{~nm})$ led to a set of fluorescing features with a strong maximum and a 
shoulder at 652 and $716 \mathrm{~nm}$ (Figure 3), respectively, which originate from the S1-S0 transition [27]. However, following excitation at the same wavelength, the emission maximum of the RGO-TPP hybrid exhibits a 93\% quenching of fluorescence emission when compared to TPP with an equal absorbance, which implies an intimate proximity between RGO and TPP as well as their subsequently strong interaction. After photoexcitation, the intramolecular donor-acceptor interaction between the RGO and TPP moieties in the RGO-TPP hybrid may include a charge transfer from the excited singlet TPP to the RGO sheet, leading to the observed fluorescence quenching and energy release, which can be explained by the extended conjugated $\pi$-system of RGO and the porphyrin mediated by a through bond mechanism, because of the unique direct linkage mode of the two moieties [28]. In addition, the fluorescence emission of RGO-TPP must be from the porphyrin moieties due to the fact that the RGO does not show a fluorescence signal.

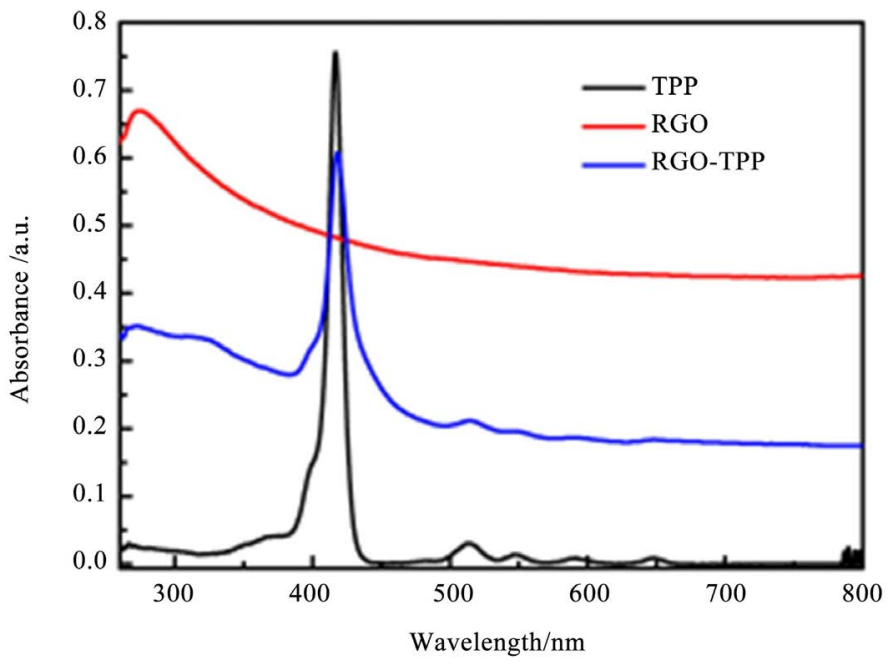

Figure 2. UV/vis absorption spectra of RGO, TPP and RGO-TPP.

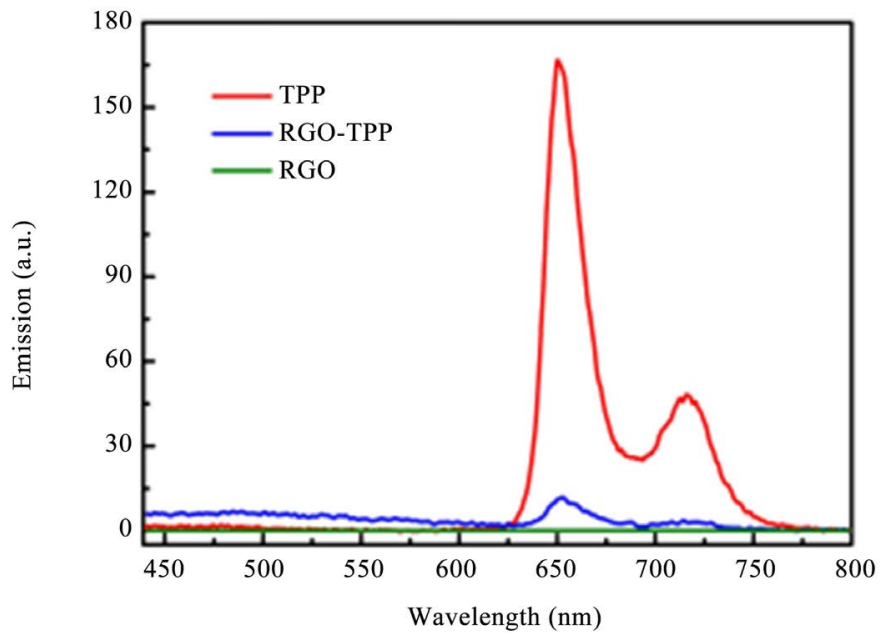

Figure 3. Fluorescence spectra of RGO-TPP, RGO and TPP in DMF, following excitation at $420 \mathrm{~nm}$. 


\subsection{Raman Spectroscopy Studies}

Raman spectroscopy reflects the significant structural changes occurring during the chemical processing from RGO to RGO-TPP (Figure 4), which indirectly confirms the success of the reaction. Upon excitation with $532 \mathrm{~nm}$ laser, the Raman spectra of all samples clearly display the $\mathrm{D}$ and $\mathrm{G}$ modes, corresponding to the structural defects (disorder-induced modes) and the first-order scattering of the E2g phonons in graphite sheets, respectively [29]. The D band usually was used to monitor the process of covalent functionalization, which transformations sp2 to sp3 sites, while the G band and the ID/IG ratio (the intensity ratio of the $\mathrm{D}$ and $\mathrm{G}$ band) were utilized to estimate the level of chemical modification [30] [31] [32]. The D and G bands of RGO are observed at 1340 and $1583 \mathrm{~cm}^{-1}$, respectively, with an ID/IG ratio of 1.20. Covalent grafting of porphyrin units onto the surface of the RGO results in the red-shifts of both the D and G bands, which located at 1352 and $1590 \mathrm{~cm}^{-1}$, respectively, with an ID/IG ratio of 1.24 .

\subsection{Microscopic Analysis}

Investigations have revealed that covalent functionalization of graphene sheets with the conjugated molecular systems can form structures with different morphologies [33]. Here we investigated the morphologies of RGO and RGO-TPP by the TEM. The TEM samples were prepared by casting a drop of sample solution onto a clean amorphous carbon-coated copper grid. From the TEM image of RGO (Figure 5(a)), the presence of transparent few-layer graphene flakes can be observed. Compared with RGO, the RGO-TPP nanohybrid exhibits an obviously different morphology (Figure 5(b)). An efficient overlapping is achieved, which in some cases lead to the formation of isolated hybrid structures. The flakes appear thicker and semitransparent with a rounded morphology which implies the presence of the $\pi-\pi$ stacking interaction [34], consistent with the observations from UV/vis and fluorescence experiments. In addition, there are some noticeable flaws on the surface of RGO-TPP (Figure 5(b)), which could be due to the presence of small flakes of porphyrins. Although information obtained from TEM should not be over-interpreted, this method still provides complementary evidence for the covalent functionalization of the RGO with the porphyrins.

\subsection{Nonlinear Absorption Performance}

Due to the efficient energy/electron transfer upon photo-excitation and the excellent third-order nonlinear optical properties of $\mathrm{C}_{60}$, graphene, carbon nanotubes and their derivatives [35] [36] [37], it would be important to study the nonlinear optical properties of RGO-TPP nanohybrids. The third-order nonlinear optical properties of materials are of great importance due to their potential applications in optoelectronic and photonic technologies. They can be used to protect human eyes and optical devices from possible damage caused by intense laser pulses. Nonlinear optical responses can arise from charge-transfer com- 
plexes because of the possibility of excited-state absorption in the dye, which can lead to reverse saturable absorption response in optical limiting [38]. In addition, rapid absorption of laser energy by the organic dye and efficient energy/electron transfer to RGO can result in the ionization of RGO nanosheets, which forms rapidly expanding microplasmas and solvent bubbles [9]. These microplasmas and solvent bubbles can scatter light from the transmitted direction, resulting in a decrease in the measured transmitted light energy. The efficiency of nonlinear scattering is mainly dependent on the clusters formations and the skeleton structure of the investigated materials [31]. The donor-acceptor system may facilitate formation of microplasmas due to the hyperpolarizabilty effect.

The third-order nonlinear optical absorption of the prepared materials was determined by the open-aperture Z-scan method at $532 \mathrm{~nm}$ with $4 \mathrm{~ns}$ laser pulses. The samples were dissolved in DMSO with a linear transmittance of $71 \%$. The open aperture Z-scan measures the normalized transmittance of the samples as it translates through the focal point of the laser pulse. At the focal point, the sample experiences maximum laser intensity, and the intensity decreases on moving away from the focus. As the sample is brought to the focal point, the nonlinear response increases and the normalized transmittance changes because of reverse saturable absorption, saturable absorption and nonlinear scattering. Figure 6 gives the Z-scan results of the normalized open aperture transmittance as a function of $\mathrm{z}$ for the prepared materials. As can be seen, the obtained open aperture Z-scan for RGO using 4 ns laser pulse was found to exhibit a transmittance maximum, indicative of saturable absorption behavior [39]. However, upon excitation with $4 \mathrm{~ns}$ laser pulses, other two materials (TPP and RGO-TPP) exhibit an inverted bell shaped transmittance valley, indicating the presence of nonlinear absorption (reverse saturable absorption) in both samples. The increase of the nonlinear optical absorption of RGO covalently functionalized with porphyrin is obviously larger than those of the individual TPP and RGO. Remarkably, the RGO-TPP has the largest dip among the transmittance curves of the studied materials [40]. This result indicates that the RGO-TPP nanohybrid has a strong nonlinear absorption; it may therefore be a promising candidate for use in optical limiting compared to the individual components (RGO and TPP) of the nanohybrid. Three mechanisms have been suggested to contribute to the nonlinear optical absorption properties of RGO nanohybrid functionalized with nonlinear-optically active chromophore: reverse saturable absorption from porphyrin, nonlinear scattering from RGO, and photo-induced energy/electron transfer from porphyrin to RGO [41]. Similar results have been observed for the nanohybrid materials of carbon nanotubes with porphyrins [42]. Comparing the nonlinear optical response of the as-prepared nanohybrid and the GO composites with metal and metal free porphyrins [12], it can be seen that the RGO-TPP possesses smaller optical nonlinearity, but different nonlinear mechanisms are believed to be responsible for the observed nonlinear performances, rendering further comments unwarranted. 


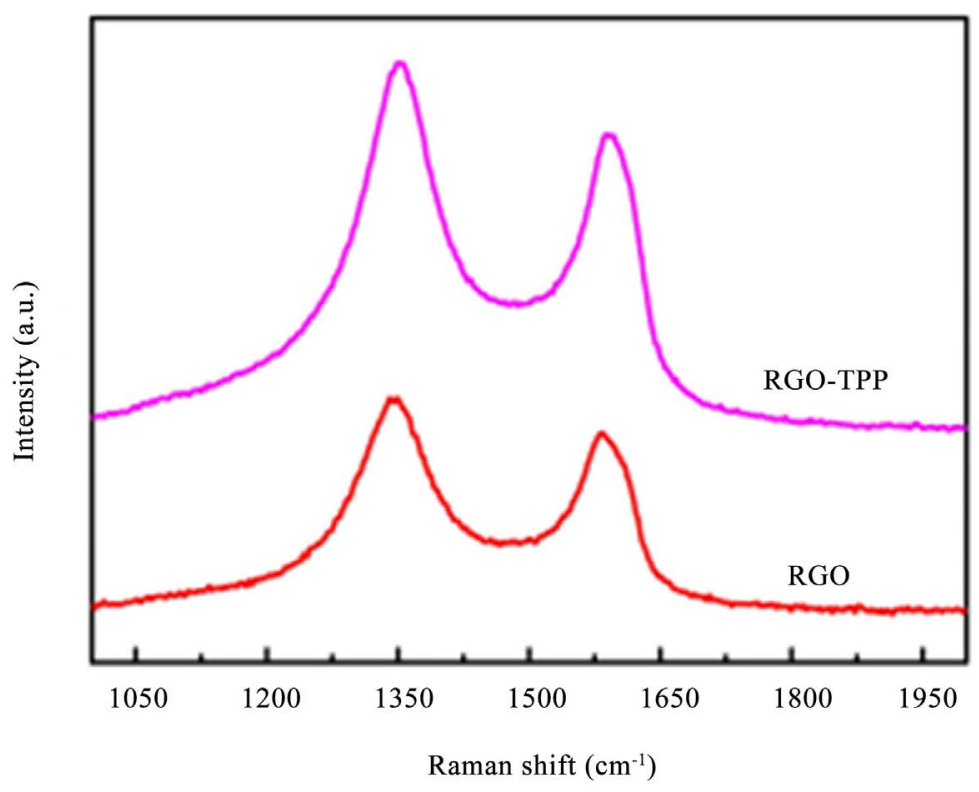

Figure 4. Raman spectra of RGO and RGO-TPP.

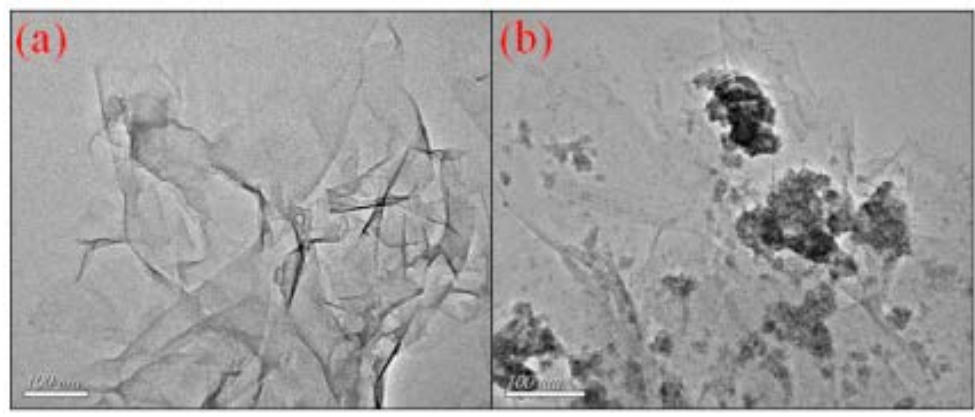

Figure 5. TEM micrograph of (a) RGO and (b) RGO-TPP.

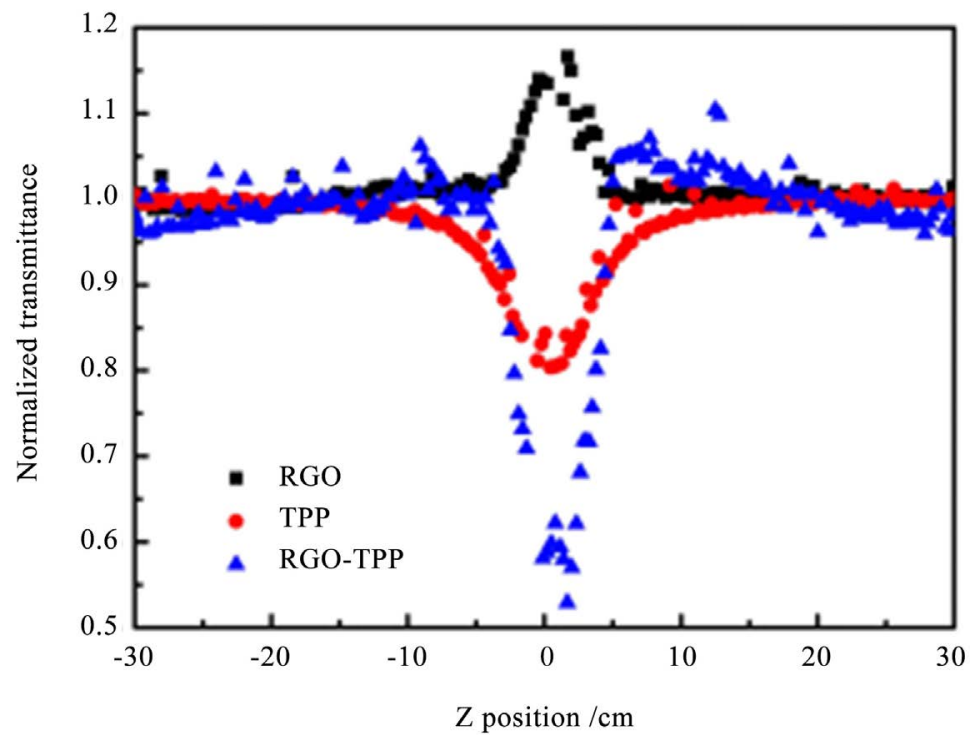

Figure 6. Open-aperture Z-scan curves of RGO, TPP and RGO-TPP nanohybrid at $532 \mathrm{~nm}$ with $4 \mathrm{~ns}$ pulse duration. 


\section{Conclusion}

In conclusion, we have reported the first covalently bonded and organic soluble RGO nanohybrid with porphyrin through free radical polymerization. The results of FTIR, UV/vis, Raman, and TEM confirm the successful fabrication of RGO-TPP nanohybrid material. For the donor-acceptor system, the fluorescence of TPP is effectively quenched by a possible photo-induced electron/energy process. As expected, upon excitation by a $532 \mathrm{~nm}$ laser of $4 \mathrm{~ns}$ and 21 ps pulses, RGO-TPP exhibits a more increased nonlinear optical absorption performance than those of individual RGO and TPP, which can be attributed to a combination of mechanisms in RGO-TPP hybrid. These results prove that the RGO-TPP nanohybrid with the unique structure and excellent electronic properties should be a good candidate for optoelectronic and nonlinear optical device applications when compared to the TPP counterparts.

\section{Conflicts of Interest}

The authors declare no conflicts of interest regarding the publication of this paper.

\section{References}

[1] Li, Y., Zhang, W., Guo, B. and Datta, D. (2017) Interlayer Shear of Nanomaterials: Graphene-Graphene, Boron Nitride-Boron Nitride and Graphene-Boron Nitride. Acta Mechanica Solida Sinica, 30, 234-240. https://doi.org/10.1016/j.camss.2017.05.002

[2] Navarro, J.J., Calleja, F., Miranda, R., et al. (2017) High Yielding and Extremely Site-Selective Covalent Functionalization of Graphene. Chemical Communications, 53, 10418-10421. https://doi.org/10.1039/C7CC04458E

[3] Geim, A.K. and Novoselov, K.S. (2007) The Rise of Graphene. Nature Materials, 6, 183-191. https://doi.org/10.1038/nmat1849

[4] Mao, S., Chang, J., Pu, H., et al. (2017) Two-Dimensional Nanomaterial-Based Field-Effect Transistors for Chemical and Biological Sensing. Chemical Society Reviews, 46, 6872-6904. https://doi.org/10.1039/C6CS00827E

[5] Xu, X., Chen, J., Luo, X., Lu, J., Zhou, H., Wu, W., Zhan, H., Dong, Y., Yan, S., Qin, J. and Li, Z. (2012) Poly(9,9'-Diheylfluorenecarbozole) Functionalized with Reduced Graphene Oxide: Convenient Synthesis Using Nitrogen-Based Nucleophiles and Potential Applications in Optical Limiting. Chemistry-A European Journal, 18, 14384-14391. https://doi.org/10.1002/chem.201201360

[6] Stankovich, S., Dikin, D.A., Dommett, G.H.B., Kohlhaas, K.M., Zimney, E.J., Stach, E.A., Piner, R.D., Nguyen, S.T. and Ruoff, R.S. (2004) Graphene-Based Composite Materials. Nature, 442,282-286. https://doi.org/10.1038/nature04969

[7] Zheng, C., Huang, L., Guo, Q.H., Chen, W.Z. and Li, W. (2018) Nonlinear Optical Responses of Carbon Quantum Dots Anchored on Graphene Oxide Hybrid in Solid-State Transparent Monolithic Silica Gel Glasses. Optics and Laser Technology, 107, 281-290. https://doi.org/10.1016/j.optlastec.2018.06.013

[8] Singla, S., Animdanso, E., Islam, A.E., Ngo, Y., Kim, S.S., Naik, R.R. and Dhinojwala, A. (2017) Insight on Structure of Water and Ice Next to Graphene Using Surface-Sensitive Spectroscopy. ACS Nano, 11, 4899-4906. 
https://doi.org/10.1021/acsnano.7b01499

[9] Remyamol, T., John, H. and Gopinath, P. (2014) Synthesis and Nonlinear Optical Properties of Reduced Graphene Oxide Covalently Functionalized with Polyaniline. Carbon, 59, 308-314. https://doi.org/10.1016/j.carbon.2013.03.023

[10] Senge, M.O., Fazekas, M., Notraras, E.G.A., Blau, W.J., Zawadzka, M., Locos, O.B. and Mhuircheartaigh, E.M.N. (2007) Nonlinear Optical Properties of Porphyrins. Advanced Materials, 19, 2737-2774. https://doi.org/10.1002/adma.200601850

[11] Dini, D., Hanack, M., Kadish, K.M., Smith, K.M. and Guilard, R. (2003) The Porphyrin Handbook. Vol. 17, Academic Press, New York, 22.

[12] Krishna, M.B.M., Venkatramaiah, N., Venkatesan, R. and Rao, D.N. (2012) Synthesis and Structural, Spectroscopic and Nonlinear Optical Measurements of Graphene Oxide and Its Composites with Metal and Metal Free Porphyrins. Journal of Materials Chemistry, 22, 3059-3068. https://doi.org/10.1039/c1jm14822b

[13] Liu, Z.B., Tian, J.G., Guo, Z., Ren, D.M., Du, F., Zheng, J.Y. and Chen, Y.S. (2008) Enhanced Optical Limiting Effects in Porphyrin-Covalently Functionalized Single-Walled Carbon Nanotubes. Advanced Materials, 20, 511-515. https://doi.org/10.1002/adma.200702547

[14] Li, P.P., Chen, Y., Zhu, J., Feng, M., Zhuang, X., Lin, Y. and Zhan. H. (2011) Charm-Bracelet-Type Poly(N-Vinylcarbazole) Functionalized with Reduced Graphene Oxide for Broadband Optical Limiting. Chemistry-A European Journal, 17, 780-785. https://doi.org/10.1002/chem.201002431

[15] Zhang, B., Liu, G., Chen, Y., Zeng, L.J., Zhu, C.X., Neoh, K.G., Wang, C. and Kang, E.T. (2011) Conjugated Polymer-Grafted Reduced Graphene Oxide for Nonvolatile Rewritable Memory. Chemistry-A European Journal, 17, 13646-13652. https://doi.org/10.1002/chem.201102686

[16] Hummers, W.S. and Offeman, R.E. (1958) Preparation of Graphitic Oxide. Journal of the American Chemical Society, 80, 1339. https://doi.org/10.1021/ja01539a017

[17] Shen, J., Hu, Y., Li, C., Qin, C. and Ye, M. (2009) Synthesis of Amphiphilic Graphene Nanoplatelets. Small, 5, 82-85. https://doi.org/10.1002/smll.200800988

[18] Fu, B., Yu, H.C., Huang, J.W., Zhao, P., Liu, J. and Ji, N.L. (2009) Mn(III) Porphyrins Immobilized on Magnetic Polymer Nanospheres as Biomimetic Catalysts Hydroxylating Cyclohexane with Molecular Oxygen. Journal of Molecular Catalysis A: Chemical, 298, 74-80. https://doi.org/10.1016/j.molcata.2008.10.015

[19] Sheik-Bahae, M., Said, A.A. and Stryland, E.W. (1989) High-Sensitivity, Single-Beam $n_{2}$ Measurements. Optics Letters, 14, 955-957. https://doi.org/10.1364/OL.14.000955

[20] Li, Z., Chen, Y., Du, Y., Wang, X., Yang, P. and Zheng. J. (2012) Triphenylamine-Functionalized Graphene Decorated with Pt Nanoparticles and Its Applications in Photocatalytic Hydrogen Production. International Journal of Hydrogen Energy, 37, 4880-4888. https://doi.org/10.1016/j.ijhydene.2011.12.045

[21] Szabó, T., Berkesi, O. and Dékány, I. (2005) DRIFT Study of Deuterium-Exchanged Graphite Oxide. Carbon, 43, 3186-3189.

https://doi.org/10.1016/j.carbon.2005.07.013

[22] Guo, Z., Du, F., Ren, D., Chen, Y., Zheng, J., Liu, Z. and Tian, J. (2006) Covalently Porphyrin-Functionalized Single-Walled Carbon Nanotubes: A Novel Photoactive and Optical Limiting Donor-Acceptor Nanohybrid. Journal of Materials Chemistry, 16, 3021-3030. https://doi.org/10.1039/B602349E

[23] Zhang, X., Hou, L., Cnossen, A., Coleman, A.C., Ivashenko, O., Rudolf, P. and Fe- 
ringa, B.L. (2011) One-Pot Functionalization of Graphene with Porphyrin through Cycloaddition Reactions. Chemistry: A European Journal, 17, 8957-8964. https://doi.org/10.1002/chem.201100980

[24] Castelaín, M., Martínez, G., Merino, P., Martín-Gago, J.Á., Segura, J.L., Ellis, G. and Salavagione, H.J. (2012) Graphene Functionalization with a Conjugated Poly(Fluorene) by Click Coupling: Striking Electronic Properties in Solution. Chemistry: A European Journal, 18, 4965-4973.

https://doi.org/10.1002/chem.201102008

[25] Cho, S., Lim, J.M., Hiroto, S., Kim, P., Shinokubo, H., Osuka, A. and Kim, D. (2009) Unusual Interchromophoric Interactions in $\beta$ Directly and Doubly Linked Corroledimmers: Prohibited Electronic Communication and Abnormal Singlet Ground States. Journal of the American Chemical Society, 131, 6412-6420. https://doi.org/10.1021/ja900220y

[26] Zhong, Q., Diev, V.V., Roberts, S.T., Antunez, P.D., Brutchey, R.L., Bradforth, S.E. and Thompson, M.E. (2013) Fused Porphyrin-Single-Walled Carbon Nanotube Hybrids: Efficient Formation and Photophysical Characterization. ACS Nano, 7, 3466-3475. https://doi.org/10.1021/nn400362e

[27] Finikova, O.S., Troxler, T., Senes, A., DeGrado, W.F., Hochstrasser, R.M. and Vinogradov, S.A. (2007) Energy and Electron Transfer in Enhanced Two-PhotonAbsorbing Systems with Triplet Cores. The Journal of Physical Chemistry A, 111, 6977-6990. https://doi.org/10.1021/jp071586f

[28] Liu, Z.B., Xu, Y.F., Zhang, X.Y., Zhang, X.L., Chen, Y.S. and Tian, J.G. (2009) Porphyrin and Fullerene Covalently Functionalized Graphene Hybrid Materials with Large Nonlinear Optical Properties. The Journal of Physical Chemistry B, 113, 9681-9686. https://doi.org/10.1021/jp9004357

[29] Gong, F., Xu, X., Zhou, G. and Wang, Z.S. (2013) Enhanced Charge Transportation in a Polyrrole Counter Electrode via Incorporation of Reduced Graphene Oxide Sheets for Dye-Sensitized Solar Cells. Physical Chemistry Chemical Physics, 15, 546-552. https://doi.org/10.1039/C2CP42790G

[30] Zhang, B., Chen, Y., Liu, G., Xu, L.Q., Chen, J., Zhu, C.X., Neoh, K.G. and Kang, E.T. (2012) Push-Pull Archetype of Reduced Graphene Oxide Functionalization with Polyfluorene for Nonvolatile Rewritable Memory. Journal of Polymer Science Part A: Polymer Chemistry, 50, 378-387. https://doi.org/10.1002/pola.25043

[31] Kavitha, M.K., John, H., Gopinath, P. and Philip, R. (2013) Synthesis of Reduced Graphene Oxide-ZnO Hybrid with Enhanced Optical Limiting Properties. Journal of Materials Chemistry C, 1, 3669-3676. https://doi.org/10.1039/c3tc30323c

[32] Paredes, J.I., Villar-Rodil. S., Solís-Fernández, P., Martínez-Alonso, A. and Tascón, J.M.D. (2009) Atomic Force and Scanning Tuning Microscopy Imaging of Graphenenanosheets Derived from Graphite Oxide. Langmuir, 25, 5957-5968.

https://doi.org/10.1021/la804216z

[33] Hsiao, M.C., Liao, S.H., Yen, M.Y., Liu, P.I., Pu, N.W., Wang, C.A. and Ma, C.C.M. (2010) Preparation of Covalently Functionalization Graphene Using Residual Oxygen-Containing Functional Groups. ACS Applied Materials \& Interfaces, 2, 3092-3099. https://doi.org/10.1021/am100597d

[34] Zhang, X., Feng, Y., Tang, S. and Feng, W. (2010) Preparation of a Graphene Oxide-Phthalocyanine Hybrid through Strong $\pi-\pi$ Interactions. Carbon, 48, 211-216. https://doi.org/10.1016/j.carbon.2009.09.007

[35] Wang, J., Hernandez, Y., Lotya, M., Coleman, J.N. and Blau, W.J. (2009) Broadband Nonlinear Optical Response of Graphene Dispersions. Advanced Materials, 21, 
2430-2435. https://doi.org/10.1002/adma.200803616

[36] Kost, A. and Tutt, L.W. (1992) Optical Limiting Performance of C60 and C70 Solutions. Nature, 356, 225-226. https://doi.org/10.1038/356225a0

[37] Liu, Z.B., Guo, Z., Zhang, X.L., Zheng, J.Y. and Tian, J.G. (2013) Increased Optical Nonlinearities of Multi-Walled Carbon Nanotubes Covalently Functionalized with Porphyrin. Carbon, 51, 419-426. https://doi.org/10.1016/j.carbon.2012.09.005

[38] Balapanuru, J., Yang, J.X., Xiao, S., Bao, Q.L., Jahan, M., Polavarapu, L., Wei, J., Xu, Q.H. and Loh, K.P. (2012) A Graphene Oxide-Organic Dye Ionic Complex with DNA-Sensing and Optical-Limiting Properties. Angewandte Chemie International Edition, 49, 6549-6553. https://doi.org/10.1002/anie.201001004

[39] Jiang, X.F., Polavarapu, L., Neo, S.T., Venkatesan, T. and Xu, Q.H. (2012) Graphene Oxides as Tunable Broadband Nonlinear Optical Materials for Femtosecond Laser Pulses. The Journal of Physical Chemistry Letters, 3, 785-790. https://doi.org/10.1021/jz300119t

[40] Song, W.N., He, C.Y., Zhang, W., Gao, Y.C., Yang, Y.X., Wu, Y.Q., Chen, Z.M., Li, X.C. and Dong, Y.L. (2014) Synthesis and Nonlinear Optical Properties of Reduced Graphene Oxide Hybrid Material Covalently Functionalized with Zinc Phthalocyanine. Carbon, 77, 1020-1030. https://doi.org/10.1016/j.carbon.2014.06.018

[41] Xu, Y.F., Liu, Z.B., Zhang, X.L., Wang, Y., Tian, J.G., Huang, Y., Ma, Y.F., Zhang, X.Y. and Chen, Y.S. (2009) A Graphene Hybrid Material Covalently Functionalized with Porphyrin: Synthesis and Optical Limiting Property. Advanced Materials, 21, 1275-1279. https://doi.org/10.1002/adma.200801617

[42] Wang, J., Chen, Y. and Blau, W.J. (2009) Carbon Nanotubes and Nanotube Composites for Nonlinear Optical Devices. Journal of Materials Chemistry, 19, 7425-7443. https://doi.org/10.1039/b906294g 